\title{
Clavipectoral plane block as a sole anesthetic technique for clavicle surgery - A case report -
}

Received July 30, 2021

Revised September 11, 2021

Accepted September 13, 2021

\section{Aileen Lagmay Rosales and Noel Singson Aypa}

Section of Regional Anesthesia and Acute Pain Management, Department of Anesthesiology, Makati Medical Center, Makati City, Philippines

Background: The clavipectoral fascial plane block was introduced by Dr. Luis Valdes in a symposium at the 2017 European Society of Regional Anesthesia and Pain Therapy Congress.

Case: Clavipectoral plane block (CPB) with intravenous sedation provided surgical anesthesia and analgesia in a 39-year-old male patient with a right midshaft clavicle fracture. This in-plane technique was used to deposit $30 \mathrm{ml}$ of a local anesthesia mixture between the clavipectoral fascia and periosteum on both the medial and lateral sides of the fracture line.

Conclusions: Excellent anesthesia and analgesia for up to $16 \mathrm{~h}$ post-block were provided by CPB during the clavicle surgery.

Keywords: Analgesia; Anesthesia; Clavicle; Local anesthesia; Nerve block.
Based on the local data provided by the Department of Health through the Online National Electronic Injury Surveillance System in 2014, only $1.64 \%$ of all recorded injuries were due to fracture of the clavicle, scapula, or humerus [1]. Moreover, most clavicle fractures (81\%) in both adults and children were located at the midshaft [2]. General anesthesia has traditionally been the preferred anesthesia for clavicle fractures because regional anesthesia via peripheral nerve block can be challenging [3]. Several case reports and series have already been published to support the use of brachial plexus block (interscalene approach) or combination blocks (interscalene with superficial cervical plexus) in clavicle surgeries. However, these techniques can be time-consuming as two separate ultrasound-guided injections are needed to provide the surgical block. Besides its ease of performance, the clavipectoral plane block (CPB) can avoid the possible adverse events associated with an interscalene block, such as ipsilateral phrenic nerve palsy, vocal cord paralysis, verte- bral artery injection, total spinal anesthesia, and pneumothorax [4].

This case report discusses the use of a clavipectoral block as the sole nerve block that provides surgical anesthesia and analgesia in a midshaft clavicle fracture. Evaluation of postoperative pain control using the Numerical Rating Scale (NRS) and opioid consumption showed the effectiveness of this plane block.

This article adheres to the applicable Enhancing the Quality and Transparency of Health Research Guidelines and Case Reports (CARE) Checklist.

\section{CASE REPORT}

The patient signed an informed consent, which stated that the clinical images taken would be used for medical teaching and in a journal publication. The Makati Medical Center Institutional Review Board (MMC IRB) approved this case

This is an Open Access article distributed under the terms of the Creative Commons Attribution Non-Commercial License (http://creativecommons.org/licenses/by-nc/4.0) which permits unrestricted non-commercial use, distribution, and reproduction in any medium, provided the original work is properly cited.

Copyright (C) the Korean Society of Anesthesiologists, 2022 
report.

A 39-year-old male patient with no known comorbidities or allergies had a closed, complete, displaced fracture in the middle third shaft of the right clavicle due to a history of trauma. The patient underwent open reduction and internal fixation of the right clavicle with plates and screws.

A peripheral nerve block was administered preoperatively under sedation with midazolam (2-5 mg) and fentanyl (50$100 \mu \mathrm{g}$ ) intravenously. Monitoring was set to 5-min intervals with supplemental oxygen at $3 \mathrm{~L} / \mathrm{min}$ via nasal cannula. In the supine position, the head was turned to the contralateral side (left). The block was administered with appropriate antisepsis. The affected clavicle was surrounded by sterile drapes. A high-frequency linear probe dressed with a sterile sonography cover was used to scan the length of the clavicle.

Fig. $1 \mathrm{~A}$ and $1 \mathrm{~B}$ show the probe positions for this block. The probe was initially placed 2 to $3 \mathrm{~cm}$ proximal to the fracture line to mark the first injection (Fig. 1A), and a similar marking was performed 2 to $3 \mathrm{~cm}$ distal to the fracture line to mark the second injection (Fig. 1B). Fig. 1C shows the sonoanatomy of the clavicle and its surrounding structures. The periosteum of the clavicle as well as the surrounding fascia were visualized for both the medial and lateral injection sites. An inplane technique was used to view the 80 -mm ultrasound-visible stimulation needle advancing in a caudad to cephalad direction until it rested on the clavipectoral fascia.

Fig. $2 \mathrm{~A}$ and $2 \mathrm{~B}$ show the needle in-plane at the medial and lateral clavicles, respectively. Aspiration was performed before the injection of local anesthesia. An injection pressure monitor attached to the syringe objectively measured the injection pressure during the administration of the peripheral nerve blocks. The total amount of local anesthetic mixture used was $30 \mathrm{ml}$ (1:1 of $0.25 \%$ levobupivacaine and 1\% lidocaine), divided into $15 \mathrm{ml}$ medial and $15 \mathrm{ml}$ lateral. The first
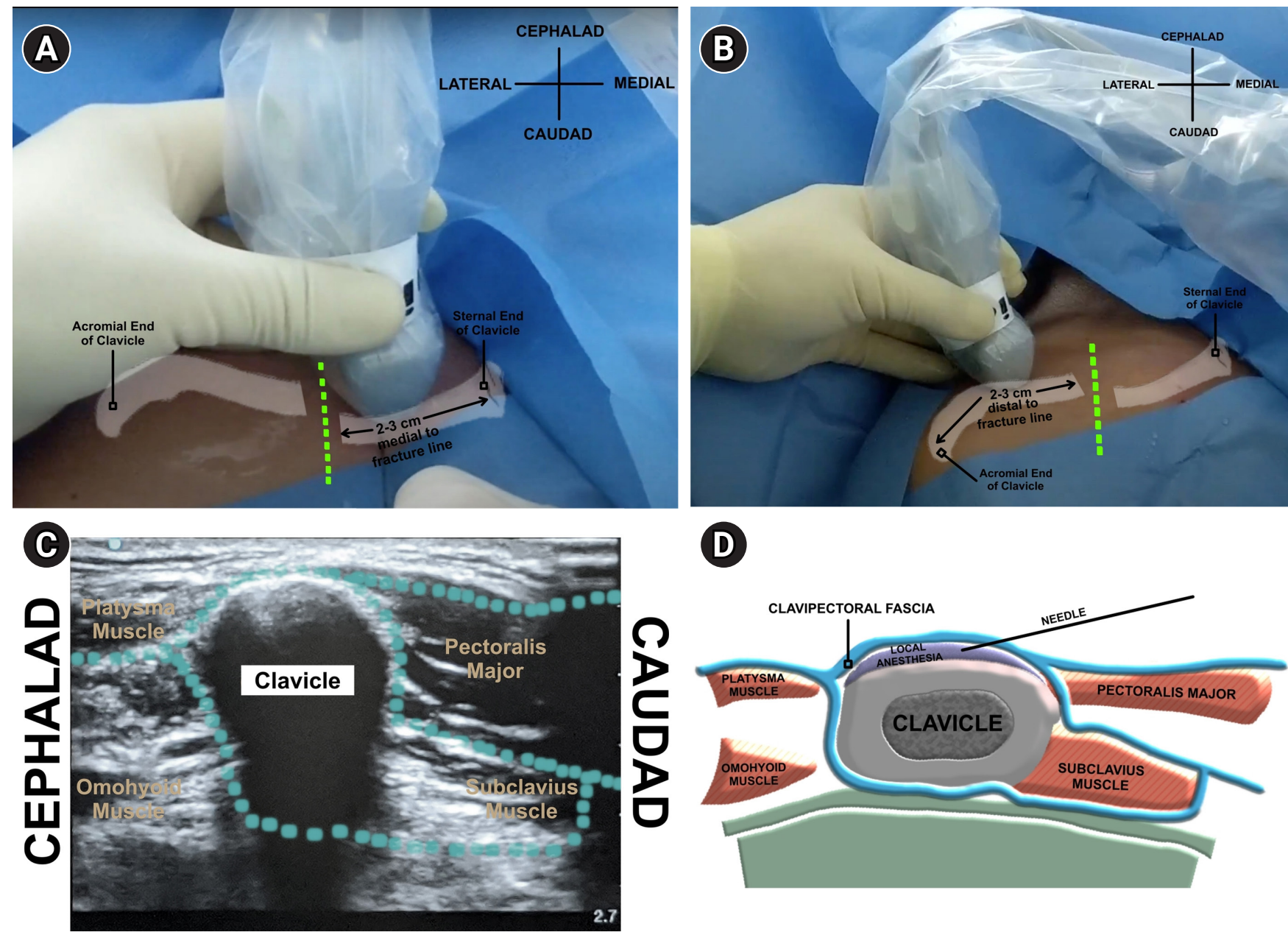

(D)

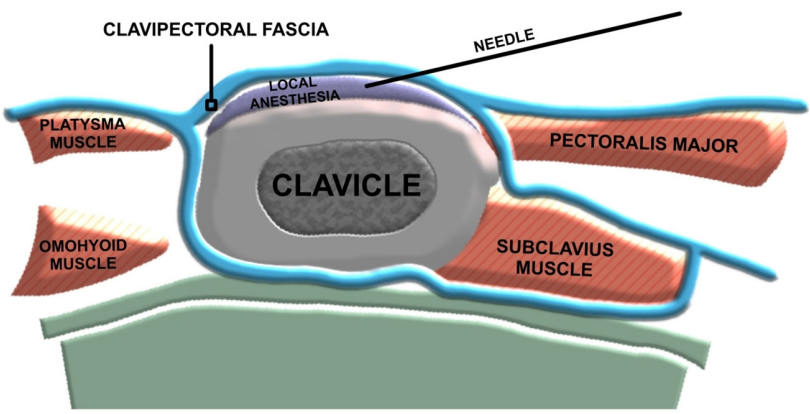

Fig. 1. (A) Scanning the clavicle medial to the fracture line. (B) Scanning the clavicle lateral to the fracture line. (C) Sonoanatomy of the clavicle and its surrounding structures (dotted lines show the clavipectoral fascia). (D) A schematic illustration showing the surrounding structures of the clavicle and the local anesthesia deposition during the clavipectoral plane block. 
injection was deposited on the medial side. The same steps were followed to block the lateral fracture line. Local anesthesia spread was observed on the medial (Fig. 2C) and lateral (Fig. 2D) sides of the clavicle.

Sensory and motor assessment of the arm and shoulder was performed 15 min after the nerve block. The right supraclavicular and infraclavicular areas were mapped for coverage of the block, which were both insensate to needle pricks. Additionally, the right upper extremity retained a full range of motion.

Sedation with dexmedetomidine $(2 \mu \mathrm{g} / \mathrm{ml})$ at $0.5-0.7 \mu \mathrm{g} /$ $\mathrm{kg} / \mathrm{h}$ was initiated before positioning the patient in a beach chair position. A Ramsey sedation score of 3 to 5 was maintained, leading to unremarkable surgery for almost two hours. The patient was monitored for pain control at the post-anesthesia care unit for two hours, with an NRS score of $0 / 10$. Twelve hours after the nerve block, the patient sat comfortably on the bed while wearing an arm sling, retaining an NRS of $0 / 10$. The right clavicle area was insensate. The patient was able to perform a range of motion over the right elbow and wrist. However, $16 \mathrm{~h}$ post-block, the patient had a pain score of NRS 7/10 on the postoperative site. It was immediately relieved by a dose of intravenous tramadol (50 $\mathrm{mg}$ ), decreasing the pain score to NRS 0/10.

The patient was administered intravenous acetaminophen (every $6 \mathrm{~h}$ for a total of three doses) and cyclooxygenase-2 inhibitor (every $12 \mathrm{~h}$ for a total of two doses) and was sent home the day after the surgery, with oral medications (non-steroidal anti-inflammatory drug with an opioid).

\section{DISCUSSION}

There is limited data on regional anesthesia for clavicle surgeries, probably due to the complex innervation of the
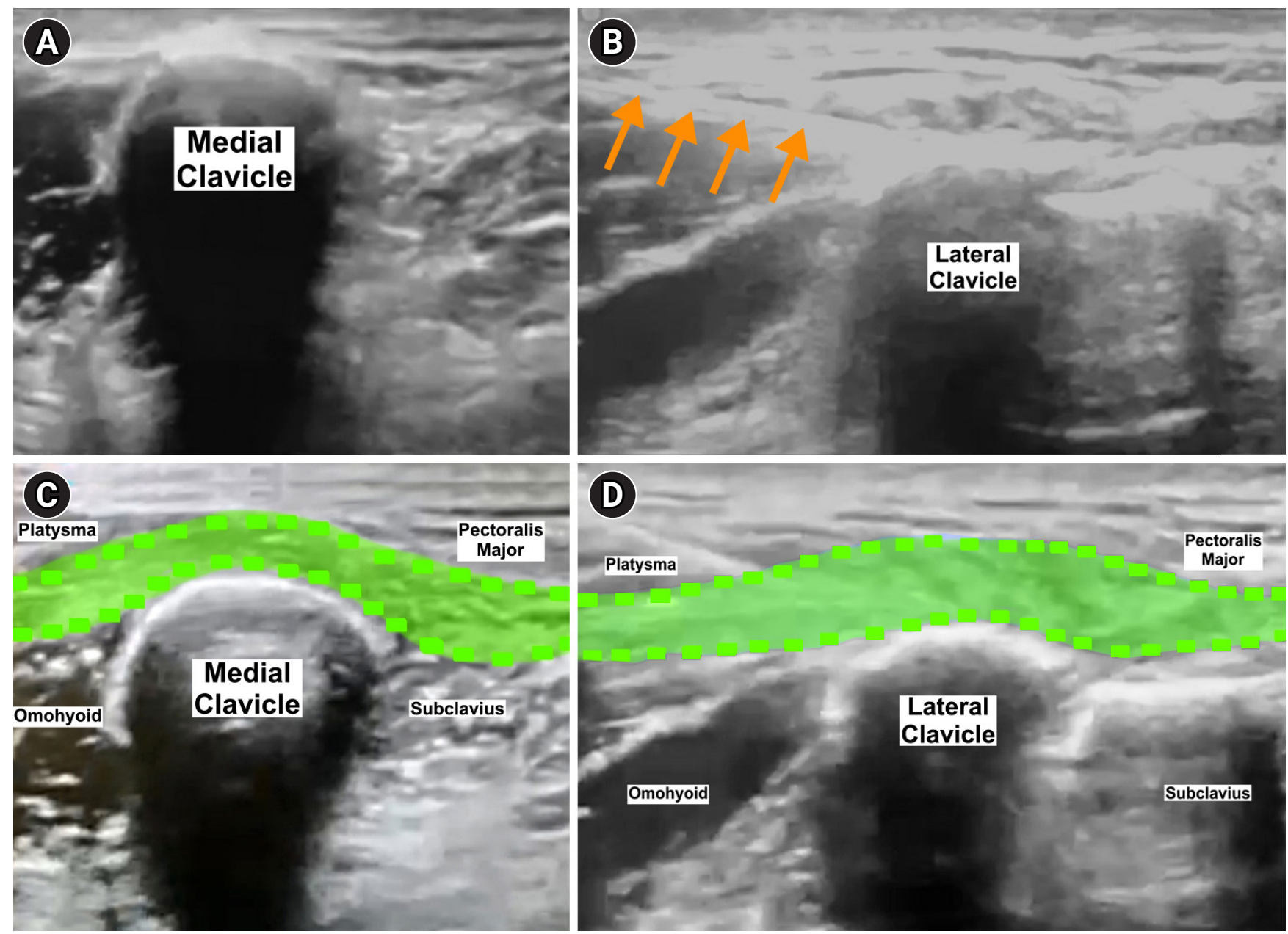

Fig. 2. (A) Needle in-plane at the medial clavicle. (B) Needle in-plane at the lateral clavicle (orange arrows show the needle). The local anesthetic spread (in green color) to the medial (C) and lateral (D) clavicle. 
clavicular region [5]. The supraclavicular nerve of the superficial cervical plexus (SCP) innervates the skin above the clavicle. However, the sensory innervation of the clavicle remains questionable. Terminal branches of the sensory nerves, such as the suprascapular, subclavian, lateral pectoral, and long thoracic nerves pass through the plane between the clavipectoral fascia and the clavicle itself. Hence, the sensory innervation of the clavicle should penetrate the clavipectoral fascia [6].

Posterior to the clavicular part of the pectoralis major muscle is a tough fascia called the clavipectoral fascia (Fig. 1D). Superiorly, this fascia splits to envelop the subclavian muscle. Medially, it connects to the first rib, before joining the fascia over the first intercostal space [7]. To form the costocoracoid ligament, the clavipectoral fascia thickens between the first rib and the coracoid process of the scapula. The structures that penetrate the clavipectoral fascia include the cephalic vein, thoracoacromial artery and vein, lymphatics, and lateral pectoral nerve. Ultimately, since this fascia envelops the clavicle, its nerve endings pierce through this structure [7].

Sensory innervation of the clavicle originates from the cervical and brachial plexuses. The chosen regional anesthesia technique should cover all the necessary innervations of the skin, muscles, and bones of the clavicle, and should correlate well with the planned surgical approach. Therefore, depositing local anesthesia between the clavipectoral fascia and periosteum may block the complete innervation [7].

Thus, the CPB has been speculated to provide effective regional anesthesia and perioperative analgesia in clavicle surgeries. However, it can only provide a surgical block if the fracture is located midshaft [6].

A recent paper by Yoshimura and Morimoto [8] presented two patients who received $\mathrm{CPB}$ after induction with general anesthesia. CPB combined with a block of the supraclavicular branch of the SCP was used to anesthetize the skin above the clavicle. CPB provided a similar analgesic effect as did the brachial plexus, but without the upper limb motor block, and the possible complication of phrenic nerve paralysis. Another recent study by Kukreja et al. [4] was conducted, wherein $\mathrm{CPB}$ was used as an adjunct peripheral nerve block. Three patients who underwent CPB preoperatively then received general anesthesia.

In this case report, a CPB with intravenous sedation provided adequate surgical anesthesia in a patient with a midshaft clavicle fracture. Instead of performing two peripheral nerve blocks (interscalene brachial plexus and superficial cervical plexus blocks), CPB provides an alternative option if either of the two mentioned blocks is contraindicated [6].

A supplemental block in the form of a "hematoma block" may be administered to cover the areas lost due to a possible break in the continuity of the fascia surrounding the fractured clavicle. Local infiltration over the subcutaneous plane of the clavicle may be provided to avoid sparing when performing CPB [7]. Neither of the two aforementioned techniques were employed in this case.

The integrity of the fascia may be lost through tissue injury and trauma caused by the surgical procedure. Hence, in fascial plane blocks, these two factors may play a major role in providing effective and successful anesthesia: integrity of the fascia and potentiality of the interfascial plane [7]. In this case, the trauma that caused the fracture might have created only a mild disruption in the fascial plane architecture, thereby not compromising the spread of the local anesthesia.

This nerve block technique may provide benefits to patients with difficult airways and in trauma patients with rib fractures and pneumothorax, where general anesthesia may increase the risk of expansion of the pneumothorax [4]. Using CPB with light sedation in this case avoided the added cost of administering general anesthesia and manipulating the airway during intubation. Further, it could gauge the coverage of the sensory and motor involvement before and after the procedure, which would have been difficult if the patient had received general anesthesia.

The midshaft location of the fracture might have also contributed to the usefulness of CPB for surgical fixation of clavicle fractures in this patient. Further studies with larger sample sizes are required to gather sufficient evidence to support the effectiveness of this novel block.

This case report supports the use of a CPB for anesthesia and analgesia in midshaft clavicle surgeries. In addition to its safety and ease of use, CPB with light sedation is a good alternative, especially if general anesthesia is not warranted.

\section{FUNDING}

None.

\section{CONFLICTS OF INTEREST}

No potential conflict of interest relevant to this article was reported. 


\section{DATA AVAILABILITY STATEMENT}

The datasets generated during and/or analyzed during the current study are available from the corresponding author on reasonable request.

\section{AUTHOR CONTRIBUTIONS}

Conceptualization: Aileen Lagmay Rosales, Noel Singson Aypa. Methodology: Aileen Lagmay Rosales, Noel Singson Aypa. Project administration: Aileen Lagmay Rosales, Noel Singson Aypa. Visualization: Aileen Lagmay Rosales, Noel Singson Aypa. Writing - original draft: Aileen Lagmay Rosales. Writing - review \& editing: Aileen Lagmay Rosales, Noel Singson Aypa. Investigation: Aileen Lagmay Rosales, Noel Singson Aypa. Supervision: Noel Singson Aypa.

\section{ORCID}

Aileen Lagmay Rosales, https://orcid.org/0000-0003-3061-5535 Noel Singson Aypa, https://orcid.org/0000-0002-6693-0011

\section{REFERENCES}

1. Rivera AS, Lam HY, Macalino JU. Epidemiology of injuries in the Philippines: an analysis of secondary data. Acta Med Philipp 2018; 52: 180-6.

2. Qin M, Zhao S, Guo W, Tang L, Li H, Wang X, et al. Open reduction and plate fixation compared with non-surgical treatment for displaced midshaft clavicle fracture: a meta-analysis of randomized clinical trials. Medicine (Baltimore) 2019; 98: e15638.

3. Shrestha BR, Sharma P. Regional anaesthesia in clavicle surgery. JNMA J Nepal Med Assoc 2017; 56: 265-7.

4. Kukreja P, Davis CJ, MacBeth L, Feinstein J, Kalagara H. Ultrasound-guided clavipectoral fascial plane block for surgery involving the clavicle: a case series. Cureus 2020; 12: e9072.

5. Fugelli CG, Westlye ET, Ersdal H, Strand K, Bjørshol C. Combined interscalene brachial plexus and superficial cervical plexus nerve block for midshaft clavicle surgery: a case series. AANA J 2019; 87: 374-8.

6. Ince I, Kilicaslan A, Roques V, Elsharkawy H, Valdes L. Ultrasound-guided clavipectoral fascial plane block in a patient undergoing clavicular surgery. J Clin Anesth 2019; 58: 125-7.

7. Sonawane K, Dixit H, Balavenkatasubramanian J, Gurumoorthi P. Uncovering secrets of the beauty bone: a comprehensive review of anatomy and regional anesthesia techniques of clavicle surgeries. Open J Orthop Rheumatol 2021; 6: 019-029.

8. Yoshimura M, Morimoto Y. Use of clavipectoral fascial plane block for clavicle fracture: two case reports. Saudi J Anaesth 2020; $14: 284-5$. 